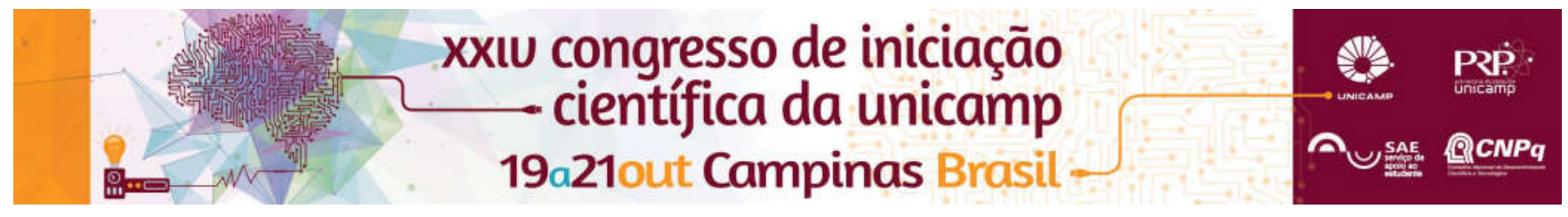

\title{
Copy-number variation of xylose isomerase for efficient adaptive evolution of engineered industrial Saccharomyces cerevisiae strain
}

\author{
João G. R. Bueno*, Marcelo F. Carazzole, Sheila T. Nagamatsu, Guilherme Borelli, Leandro V. Santos, Gonçalo \\ A. G. Pereira.
}

\begin{abstract}
The development of yeast strains capable of fermenting a five-carbon sugar xylose is a key step for achieving a feasible production of second-generation ethanol. An efficient expression of xylose isomerase (xylA), the primary gene of xylose metabolism, is essential in order to achieve an efficient conversion of this sugar. To enhance strain performance following initial genetic engineering steps, cycles of adaptive evolution are typically conducted in xylose medium. However, this process can be slow as it depends on xylA activity. In this study, we established a faster and powerful process for evolution based on the increased copy-number of xylA. The cell that presents higher copy-number of this gene during adaptive evolution in xylose media could convert this sugar and produce ethanol faster, being positively selected by evolution.
\end{abstract}

\section{Key words: \\ Copy-number variation, adaptive evolution, xylose isomerase}

\section{Introduction}

The demand for alternative and renewable sources of energy has increase the importance on make the second-generation $(2 \mathrm{G})$ ethanol technology economically viable ${ }^{1}$. 2G-ethanol production use lignocellulosic biomass as primary source, extracting sugars from cellulose and hemicellulose chains of the plant cell wall ${ }^{2}$. The lignocellulosic biomass is rich in fivecarbon sugar xylose, which cannot be fermented by Saccharomyces cerevisiae. ${ }^{2}$

Accordingly, it is essential for the $2 G$ ethanol industry the development of a genetic modified $S$. cerevisiae capable of efficiently convert xylose into ethanol. The enzyme xylose isomerase ( $x y l A$ gene) can convert xylose to xylulose, a secondary metabolite that can be fermentable by $S$. cerevisiae through pentose phosphate pathway (PPP) to ethanol. However, the $x y / A$ activity in $S$. cerevisiae is very low, requiring a larger number of copies of this gene in order to improve the enzyme activity. A usually process called adaptive evolution is a required step to allow a rapid xylose fermentation

The primary aim of this study is to establish a faster xylose adaptive evolution process by using a strategy to increase the copy-number of xylose isomerase stable integrated in genome.

\section{Results and Discussion}

In this study, we developed a plasmid named of pOXyIATy1 that contains the xylA gene, under the control of a strong and constitutive promoter and terminator of $T D H 1$, flanked by two long-terminal repeats (LTR) from retrotransposon Ty1 in the same direct orientation. LEU2 was used as the selection marker.This plasmid was digest with the restriction enzyme BamH1. The linear sequence obtained was used for the transformation of an industrial S. cerevisiae strain named LV27. The selection markers and PCR approach confirmed the positive transformants.
Given the considerable abundance of Ty elements in the genome of $S$. cerevisiae, it is expected that insertion could take place in more than one site by homologous recombination. Another possibility is the formation of an unequal crossing between sisters chromatids during cell division ${ }^{3}$. The LTR sequences in the same orientation can recombine resulting in migration of one element and exponential increasing of $x y / A$ in one of the individuals. In both possibilities, the cells that inherit higher copy number of $x y I A$ tend to be positively selected by evolution.

Our preliminary results show that after transformation with pOXyIATy1, in a very short time of evolution ( $<10$ batch cultivations), we are able to select cells with improved fermentative fitness. After genome sequencing, the comparison of read-depth between parental and evolved strains revealed a substantial amplification of a region containing the $x y l A$ gene. This region was amplified $>10$-fold among the evolved selected strains during evolution.

\section{Conclusions}

Currently, based on these preliminary results, we are developing a new and more efficient strategy of evolution, based on yeasts that contain a high copynumber of $x y l A$, the key gene of xylose metabolism. This process makes possible the development of yeast strains capable of being utilized in second-generation ethanol production.

\section{Acknowledgement} and $\mathrm{CNPq}$

We thank the financial support of Finep, BNDES

\footnotetext{
References:

1. Vitousek, P. M., Mooney, H. a, Lubchenco, J. \& Melillo, J. M. Human Domination of Earth 's Ecosystems. Science (80-. ). 277, 494-499 (1997).

2. Santos, L. V. et al. Second-Generation Ethanol: The Need is Becoming a Reality. Ind. Biotechnol. 12, 1-18 (2016).

3. Zhang, H. et al. Gene Copy Number Variation in Haploid and Diploid Strains of the Yeast. Genetics 193, 785-801 (2013).
} 